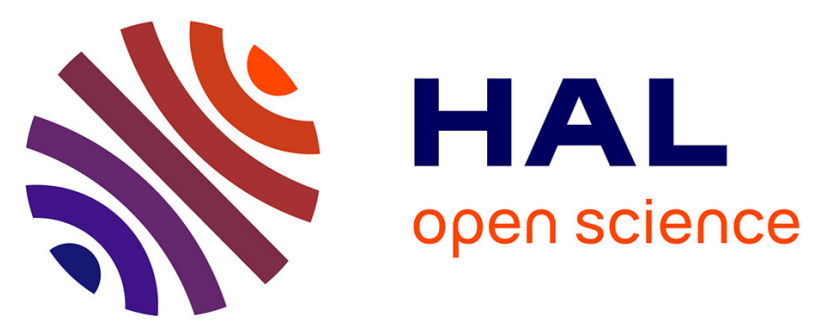

\title{
Stabilizing Heteroscedastic Noise With the Generalized Anscombe Transform. Application to Accurate Prediction of the Resolution in Displacement and Strain Maps Obtained With the Grid Method.
}

Michel Grediac, Frédéric Sur

\section{To cite this version:}

Michel Grediac, Frédéric Sur. Stabilizing Heteroscedastic Noise With the Generalized Anscombe Transform. Application to Accurate Prediction of the Resolution in Displacement and Strain Maps Obtained With the Grid Method.. SEM Annual Conference \& Exposition on Experimental and Applied Mechanics - 2014, Jun 2014, Greenville, SC, United States. pp.225-230, 10.1007/978-3-31906986-9_25. hal-01001869

\section{HAL Id: hal-01001869 \\ https://inria.hal.science/hal-01001869}

Submitted on 12 Sep 2014

HAL is a multi-disciplinary open access archive for the deposit and dissemination of scientific research documents, whether they are published or not. The documents may come from teaching and research institutions in France or abroad, or from public or private research centers.
L'archive ouverte pluridisciplinaire HAL, est destinée au dépôt et à la diffusion de documents scientifiques de niveau recherche, publiés ou non, émanant des établissements d'enseignement et de recherche français ou étrangers, des laboratoires publics ou privés. 


\title{
STABILIZING HETEROSCEDASTIC NOISE WITH THE GENERALIZED ANSCOMBE TRANSFORM. APPLICATION TO ACCURATE PREDICTION OF THE RESOLUTION IN DISPLACEMENT AND STRAIN MAPS OBTAINED WITH THE GRID METHOD
}

\author{
M. Grédiac ${ }^{1}$, F. Sur ${ }^{2}$ \\ ${ }^{1}$ Clermont Université, Université Blaise Pascal, Institut Pascal, UMR CNRS 6602 \\ BP 10448, 63000 Clermont-Ferrand, France \\ ${ }^{2}$ Laboratoire Lorrain de Recherche en Informatique et ses Applications, UMR CNRS 7503 \\ Université de Lorraine, CNRS, INRIA projet Magrit, Campus Scientifique, BP 239 \\ 54506 Vandoeuvre-lès-Nancy Cedex, France
}

\begin{abstract}
The objective of this paper is to show that it is possible to predict the noise level in displacement and strain maps obtained with the grid method, but that actual noise of camera sensors being heteroscedastic, it is necessary to stabilize this noise in grid images prior to employing the predicting formulas. The procedure used for this purpose relies on the Generalized Anscombe Transform. This transform is first described. It is then shown that experimental and theoretical resolutions in strain maps obtained with the grid method are in good agreement when this transform is employed.
\end{abstract}

Keywords: displacement, Generalized Anscombe Transform, grid method, noise, strain measurement, metrological performance

\section{INTRODUCTION}

Assessing the metrological performance of full-field measurement techniques is a key-issue in experimental mechanics. In this context, this paper deals with the a priori determination of the resolution in displacement and strain maps obtained with the grid method, the spatial resolution being fixed by the grid image processing procedure. Two points are addressed more specifically:

1- closed-form formulas which provide the link between resolution, spatial resolution and standard deviation of sensor noise in grid images taken by a camera are given and discussed. A specific predictive formula is obtained for both the displacement and for strain maps;

2- experiments are carried out on real grid images to check whether these formulas are verified.

As in most of the studies discussed in the literature dealing with noise prediction in displacement and strain maps obtained with various full-field measurement techniques, the closed-form formulas mentioned in point 1 above are obtained here assuming that noise in the grid images is white. This means that its characteristics (especially its variance) are constant throughout the grid images. In statistics, such a noise is referred to as homoscedastic. 
The actual response of camera sensors is however not homoscedastic since its standard deviation significantly depends on brightness at any pixel [1], and the latter obviously changes from one pixel to another in any contrasted image. Thus actual noise in images grabbed by cameras is not homoscedastic but heteroscedastic. This leads any predictive formulae for the noise in final displacement or strain maps obtained under the assumption of homoscedastic noise not to be satisfied in practice, or to be at least more or less severely impaired by too rough this assumption.

Stabilizing the changing variance of a heteroscedastic noise, thus transforming a heteroscedastic noise into a homoscedastic one, is theoretically possible when this noise is e.g. Poisson-Gaussian. This specific transform is the so-called Generalized Anscombe Transform [2]. This transform involves some noise parameters of the camera which need therefore to be identified.

The grid method, which is employed here to retrieve displacement and strain maps, is first briefly presented. The main steps of the GAT are then described. It is finally applied to a set of real images grabbed by a CCD camera. Other phenomena may corrupt images such as microvibrations or light flicker. They are eliminated using another transform which is detailed in another paper [3]. It is finally shown through experiments that applying these transforms to actual grid images leads the abovementioned formulas to be very satisfactorily verified, thus illustrating that noise in final displacement and strain maps can be accurately and reliably predicted.

\section{OBTAINING DISPLACEMENT AND STRAIN MAPS THE WITH GRID METHOD}

The grid method consists first in depositing a crossed grid on the surface under investigation in order to track the change in the geometry of the grid while loading increases, and to deduce the $2 \mathrm{D}$ strain fields from these images. The typical value of the pitch (denoted $\mathrm{p}$ ) is $0.2 \mathrm{~mm}$. The procedure for depositing the grids is given in [4].

Processing grid images consists first in extracting the phases along directions $\mathrm{x}$ and $\mathrm{y}$ both in the reference and in the current images. Phase extraction is carried out with the Windowed Fourier Transform (WFT) [5], in which the envelope considered in the present study is Gaussian, as in [6]. The displacement is obtained from the phase changes between current and reference grid images using the following equation:

$$
\mathrm{u}_{\mathrm{i}}=-\frac{\mathrm{p}}{2 \pi} \Delta \Phi_{\mathrm{i}}, \mathrm{i}=\mathrm{x}, \mathrm{y}
$$

Thus

$$
\varepsilon_{\mathrm{ij}}=-\frac{\mathrm{p}}{4 \pi} \Delta\left(\frac{\partial \Phi_{\mathrm{i}}}{\partial \mathrm{x}_{\mathrm{j}}}+\frac{\partial \Phi_{\mathrm{j}}}{\partial \mathrm{x}_{\mathrm{i}}}\right), \mathrm{i}, \mathrm{j}=\mathrm{x}, \mathrm{y}
$$

\section{PREDICTIVE FORMULAS}

After pioneering work in [7], Ref. [8] gives a closed-form expression for the variance of the noise in the phase or the phase derivative images from the variance of the noise in the grid image. These expressions are obtained assuming that:

- the grid images are corrupted by a homoscedastic Gaussian white noise of constant variance;

- $\quad$ the grid used as a spatial carrier does not feature any defect such as pitch variation, so the noise in the maps is only due to sensor noise;

- the envelope of the kernel used to extract the phase and phase derivative maps is a Gaussian function. Its standard deviation is denoted $\sigma$.

In [8], this result was establish to feed the Wiener filter used to deconvolve strain images, as in [9]. These predictive equations for the variance can easily be rewritten as links between resolution, spatial resolution, standard deviation of noise in the grid images, and various parameters characterizing the grid profile.

Resolution can be defined by the "change in quantity being measured that causes a change in the corresponding indication greater than one standard deviation of the measurement noise" [10]. Note that this quantity is not defined by any standard, so any multiple of one standard deviation could be considered: this would only change the equations given below. Spatial 
resolution is defined here by the shortest distance between two spatially independent measurements. Since a Gaussian window is used in the approach employed here, it is reasonable to define this quantity by a multiple of the standard deviation $\sigma$ of the Gaussian window, say $6 \sigma$ according to the three-sigma rule.

The links between all these quantities are as follows [11]

$$
\left\{\begin{array}{l}
\sigma_{\Phi} \times \mathrm{d}_{\Phi}=\frac{3}{\sqrt{2 \pi}} \times \frac{\mathrm{a}}{\mathrm{K}} \times \sigma_{\mathrm{im}} \\
\sigma_{\Phi^{\prime}} \times \mathrm{d}_{\Phi^{\prime}}^{2}=\frac{9}{\sqrt{\pi}} \times \frac{\mathrm{a}}{\mathrm{K}} \times \sigma_{\mathrm{im}}
\end{array}\right.
$$

where:

- $\sigma_{\Phi}$ is the resolution in the phase map as defined above;

- $\quad \mathrm{d}_{\Phi}$ is the spatial resolution in the phase map as defined above;

- $\quad \mathrm{a}$ is the area of one pixel. Dimensions being given in pixels, $\mathrm{a}=1$ in the following;

- $\quad \mathrm{K}$ is the modulus of the WFT used to extract the phase;

- $\sigma_{\mathrm{im}}$ is the standard deviation of the sensor noise assumed here to be homoscedastic.

Similar equations are deduced for both the displacement and the strain:

$$
\left\{\begin{array}{l}
\sigma_{\mathrm{u}} \times \mathrm{d}_{\mathrm{u}}=\mathrm{p} \frac{3}{2 \pi^{3 / 2}} \times \frac{\mathrm{s}}{\mathrm{K}} \times \sigma_{\mathrm{im}} \\
\sigma_{\varepsilon} \times \mathrm{d}_{\varepsilon}^{2}=\mathrm{p} \frac{9 \sqrt{2}}{2 \pi^{3 / 2}} \times \frac{\mathrm{s}}{\mathrm{K}} \times \sigma_{\mathrm{im}}
\end{array}\right.
$$

where:

- $\sigma_{\mathrm{u}}$ is the resolution in the displacement map as defined above;

- $\quad \mathrm{d}_{\mathrm{u}}$ is the spatial resolution in the displacement map as defined above;

- $\quad \mathrm{p}$ is the pitch of the grid deposited on the specimen.

Several remarks can be drawn from these equations:

- Equations (3-a) and (4-a) show that the metrological performance of the phase and the displacement determination are characterized by a relationship similar to that deduced from the Heisenberg uncertainty principle [12] in the sense that the resolution and spatial resolution cannot be simultaneously arbitrarily low;

- differentiation involved in the calculation of the phase derivative and strain components causes the spatial resolution to be squared in the left-hand side of Equations (3-b) and (4-b). Consequently, improving the spatial resolution by a factor 3 impairs the resolution by a factor 9. A similar property has already been discussed for the global performance of DIC in [13] for instance;

- both products are proportional to the standard deviation of the noise $\sigma_{\mathrm{im}}$ in the grid image, which seems quite logical. They are also inversely proportional to the modulus $\mathrm{K}$ of the WFT of the grid image. This modulus can easily be obtained at any pixel of the grid image since the WFT is calculated for the determination of the phase anyway. $\mathrm{K}$ is actually proportional to three quantities [11]: the brightness, the contrast of the grid and the first coefficient of the development of the grid profile in Fourier series, denoted $d_{1}$ here. Finding that noise is all the more magnified as brightness and contrast are low is logical. $d_{1}$ is maximum for a sine profile, so any printed grid should have a profile tending to a sine to minimize noise sensor propagation. If rectangular profile are employed, the black and white lines should at least have the same width [11];

- $\quad$ it is finally worth mentioning that in theory, the phase distribution retrieved by the WFT does not depend on the nature of the line profile of the grid (ignoring the ) whereas the noise level in the displacement map depends on this profile. The same remark holds for the displacement. 


\section{NOISE MODEL AND NOISE NORMALIZATION WITH THE GENERALIZED ANSCOMBE TRANSFORM}

A classic model for the camera response function is to consider that it is linear. It means that the grey level measured at a pixel is proportional to the quantity of light photons arriving at a CCD sensor cell, plus a dark current [1]:

$$
\mathrm{s}(\mathrm{x}, \mathrm{y})=\mathrm{g} \eta_{\mathrm{p}(\mathrm{x}, \mathrm{y})}+\delta(\mathrm{x}, \mathrm{y})
$$

where $s$ is the observed signal and $\mathrm{g}$ is the gain of the electronic system. $\eta_{\mathrm{p}(\mathrm{x}, \mathrm{y})}$ is the number of collected light photons at a pixel $(\mathrm{x}, \mathrm{y})$. This is a random variable which follows a Poisson distribution of parameter $\mathrm{p}(\mathrm{x}, \mathrm{y})$. This parameter is assumed to be spatially independently distributed. $\delta(\mathrm{x}, \mathrm{y})$ models the dark current and readout noise. This is a Gaussian white noise of mean $\mu_{\mathrm{d}}$ and variance $\sigma_{\mathrm{d}}^{2} \cdot \eta_{\mathrm{p}(\mathrm{x}, \mathrm{y})}$ and $\delta(\mathrm{x}, \mathrm{y})$ being assumed to be independent, we can deduce the following linear relation between the expectation $\mathrm{E}(\mathrm{s}(\mathrm{x}, \mathrm{y}))$ and the variance $\operatorname{Var}(\mathrm{s}(\mathrm{x}, \mathrm{y}))$ of $\mathrm{s}(\mathrm{x}, \mathrm{y})$ :

$$
\operatorname{Var}(\mathrm{s}(\mathrm{x}, \mathrm{y}))=\mathrm{gE}(\mathrm{s}(\mathrm{x}, \mathrm{y}))+\sigma_{\mathrm{d}}^{2}-\mathrm{g} \mu_{\mathrm{d}}
$$

The noisy signal is therefore a heteroscedastic random variable whose variance linearly depends on its expectation, thus illustrating that noise is heteroscedastic. This heteroscedastic noise can be changed into a homoscedastic one by using the GAT introduced above [1]. This transform stabilizes to 1 the variance of any Poisson-Gaussian variable following the model given in Equation (6). The GAT writes as follows:

$$
\operatorname{GAT}(\mathrm{s}(\mathrm{x}, \mathrm{y}))=\frac{2}{\mathrm{~g}} \sqrt{\mathrm{gs}(\mathrm{x}, \mathrm{y})+\frac{3}{8} \mathrm{~g}^{2}+\sigma_{\mathrm{d}}^{2}-\mathrm{g} \mu_{\mathrm{d}}}
$$

In practice, the parameters involved in the GAT are those driving the linear equation between variance and expectation given in Equation (6). In theory, they can be found either from one single image, as in [1] for instance, or by considering a stack of images of the same static scene (here a grid), calculating the mean and variance of noise at any pixel, plotting the corresponding point cloud in the variance-mean plane and finally fitting the data by a straight line whose slope and intercept are $\mathrm{g}$ and $\sigma_{\mathrm{d}}^{2}-\mathrm{g} \mu_{\mathrm{d}}$, respectively. Note that $\mathrm{g}, \sigma_{\mathrm{d}}$ and $\mu_{\mathrm{d}}$ are not always given in datasheets of cameras, it therefore necessary to identify them using a dedicated procedure as in Ref. [3] for instance.

\section{EXPERIMENTAL RESULTS}

The objective here is to check whether the above theoretical predictions are satisfied in practice and to observe the benefit of the GAT on grid images grabbed by a CCD sensor. The brightness being not constant throughout any grid image and the spatial resolution being fixed, it is worth mentioning, by considering Equations (3)-(4) above, that the resolution is not a constant quantity: it varies throughout the maps since it is inversely proportional to the brightness which is in general heterogeneous. Equations (3)-(4) must therefore be verified at any pixel of a stack of grid images.

Finding a state of reference for a strain distribution is an issue. A mere translation test was therefore performed to check the abovementioned equations, as in [14] for instance, the strain distribution being theoretically null in this case. In this study, the specimen was an aluminum rectangular plate on which a grid was transferred using the technique described in [4]. The pitch of the grid was $0.2 \mathrm{~mm}$ and the distance between camera and specimen was such that 5 pixels were employed to digitize a grid pitch. The grid was lit by three flexible and movable light guides fed by a cold light source. 200 grid images of this grid image were grabbed before and after translating the specimen by $0.1 \mathrm{~mm}$. A SENSICAM QE camera featuring a 12-bit 1040×1376-pixel CCD sensor was used for this purpose. The mean value and standard deviation for the temporal distribution of gray level in grid images can be easily obtained pixelwise from this stack of images. Phase, phase derivative, displacement and strain in the corresponding maps can also be easily calculated at any pixel of the maps extracted from these images, and then compared to their theoretical predictions.

Heteroscedastic noise in the grid images is illustrated in Figure 1-a which represents a close-up of the standard deviation of the gray level distribution. In this figure, the size of each pixel is $40 \times 40$ micrometers ${ }^{2}$. 


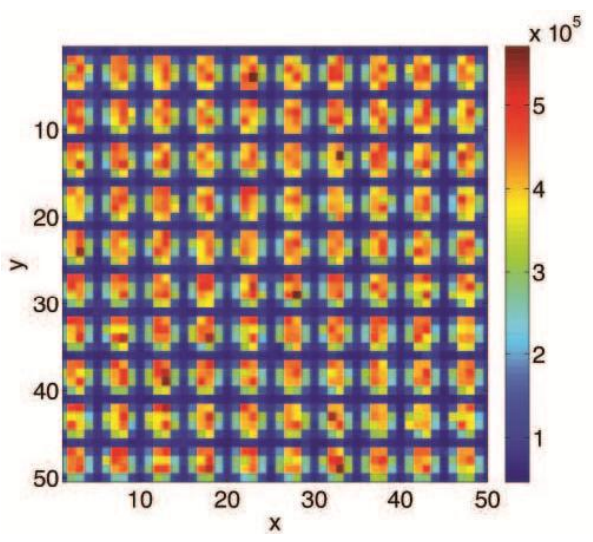

a- variance of the noise

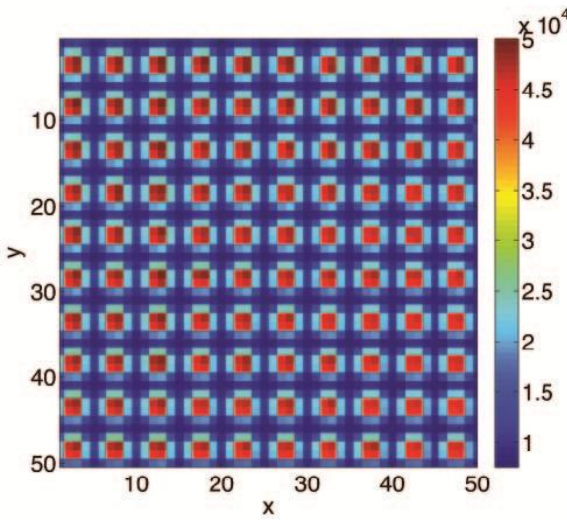

b- gray level distribution

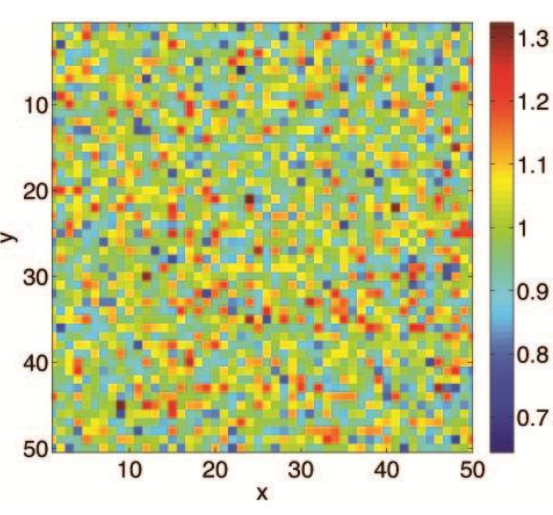

c- normalized variance obtained

after eliminating microvibrations and

applying the GAT

Figure 1: Benefit of the GAT. Close-up of a mean grid image and corresponding spatial variance distributions

The grid pattern is easily recognizable by comparing this map with a close-up of the same zone in the grid (see Figure 1-b). More precisely, the standard deviation of the gray level distribution is all the greater as the gray level is high.

The GAT has been applied to normalize noise throughout the grid images. Parameters involved in Equation (7) were first identified, so the GAT could then be applied to normalize noise. It can then be checked that the standard deviation of the gray level distribution at any pixel is randomly distributed around one, as illustrated in Figure 1-c. It must be pointed out that unavoidable microvibrations that occurred during grid image grabbing (in a laboratory context) significantly corrupted this noise characterization despite their tiny amplitude of some micrometers. This effect can be detected in Figure 1-a: the bright center of all the cells of the grid pattern is slightly upwardly stretched since microvibrations occur along the vertical direction. A specific procedure described in [3] was therefore employed to get rid this effect before applying the GAT.

Equations 4 are then employed to predict the resolution in displacement and strain maps. Since the GAT has been applied, $\sigma_{\text {im }}$ is equal to one in these equations. In the same way, $\mathrm{K}$ is the modulus of the normalized images, not that of the initial raw images. The comparison between observed and predicted resolutions along the vertical direction is shown in Figure 2 as an example. It can also be checked that the mean value of the strain at any pixel is very close to zero [11].

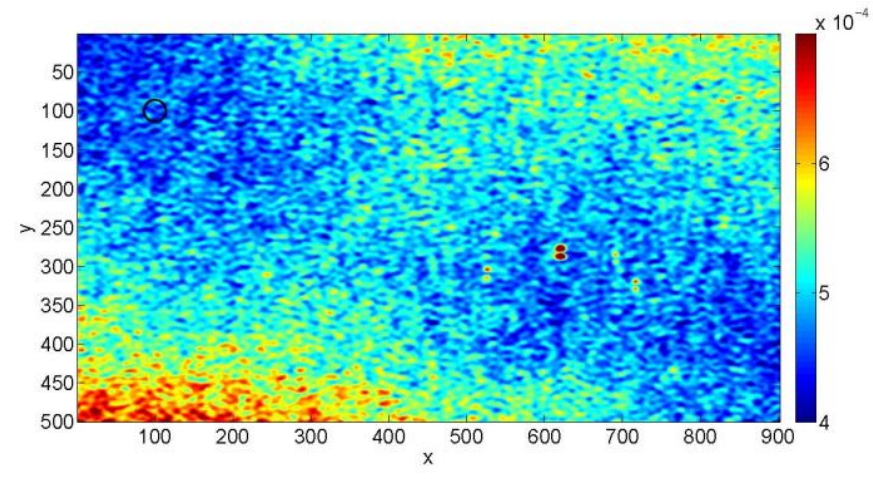

a- Observed with a stack of 200 strain maps

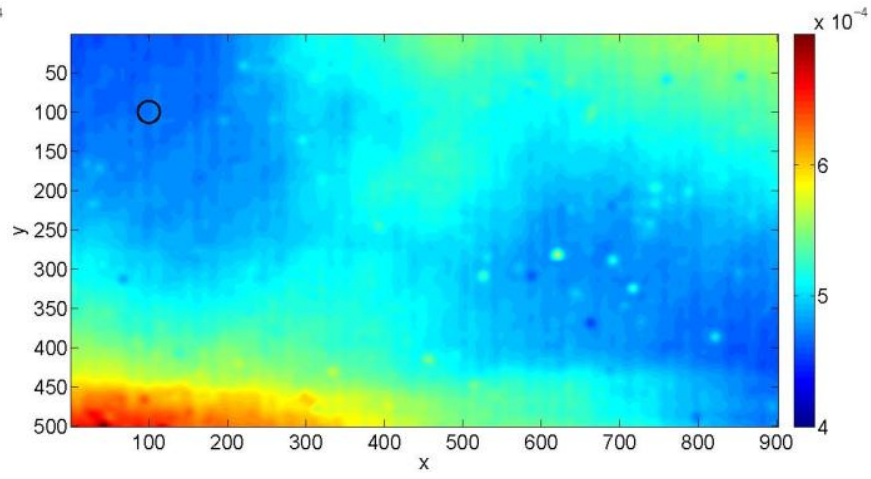

b-Predicted with Equation (4-a)

Figure 2: Resolution in the strain map

Several remarks can be drawn from this figure:

- the global aspect of the experimental and theoretical distributions of the resolution are very similar. The small blobs visible in the experimental maps are due to noise correlation that is not accounted for in Equation (4-a). The next step will be to check whether it corresponds to noise correlation predicted in Ref. [8];

- a slight vertical pattern as well as some isolated outliers are visible in the predicted map. They are due to grid printing defects; 
- the resolution is not constant throughout the map. This is due to the non-uniform lightning. By comparing this distribution directly with the gray level of the grid image or the distribution of the modulus of the WFT, it can be easily checked that the noise level is all the lower as the brightness is high. It is worth mentioning that obtaining a uniform lighting is tricky in practice: even though the grid image observed to the naked eye on the screen of the companion PC of the camera could appear to be uniform (as in the current example);

- observed and predicted distributions for the displacement are also in very good agreement [11] (not shown here);

- a black circle is superposed at the top left of each map. The diameter is equal to the spatial resolution, considered here to be equal to $6 \times \sigma=30$ pixels. The reader can see at a glance the metrological performance of the technique for retrieving the strain: the circle provides the spatial resolution which is constant throughout the map while the resolution changes from one point to each other because the brightness changes throughout the grid images in this example. Impairing the spatial resolution in the strain map (thus enlarging the circle) would cause the noise level to descrease and the map to be more blurred, the observed distribution being equal to the actual one convolved by the envelope of the kernel of the WFT [8].

\section{CONCLUSION}

Closed-form expressions are given to predict how noise propagates between camera sensor and displacement and strain maps obtained with the grid method. Noise of camera sensor being heteroscedastic, it must first be stabilized with the GAT to obtain experimental results in very good agreement theoretical expectations. reliably estimating the noise level distribution in strain maps is crucial, not only to assess the metrological performance of the grid method as a tool for measuring strain maps, but also to perform efficient deconvolution of the strain maps to retrieve the actual strain distributions [6].

\section{REFERENCES}

[1] G.E. Healey, R. Kondepudy. Radiometric CCD camera calibration and noise estimation. IEEE Transactions on Pattern Analysis and Machine Intelligence, 16(3):267-276, 1994

[2] F. Murthagh, J.L. Starck, and A. Bijaoui. Image restoration with noise suppression using a multiresolution support. Astronomy and astrophysics, 112:179-189, 1995.

[3] F. Sur, M. Grédiac, Sensor noise modeling by stacking pseudo-periodic grid images affected by vibrations, IEEE Signal Processing letters, 21(4): 432-436, 2014

[4] J.L. Piro, M. Grédiac, Producing and transferring low-spatial-frequency grids for measuring displacement fields with moiré and grid methods, Experimental Techniques, 28(4): 23-26, 2004

[5] Y. Surrel. Photomechanics, Topics in Applied Physic 77, chapter on fringe analysis, pp. 55-102. Springer, 2000.

[6] C. Badulescu, M. Grédiac, and J.-D. Mathias. Investigation of the grid method for accurate in-plane strain measurement. Measurement Science and Technology, 20(9):095102, 2009

[7] Y. Surrel Additive noise effect in digital phase detection,Applied Optics, 36(1): 271-276, 1997

[8] F. Sur, M. Grédiac. Towards deconvolution to enhance the grid method for in-plane strain measurement. Inverse Problems and Imaging, American Institute of Mathematical Sciences, 8(1): 259-291, 2014

[9] M. Grédiac, F. Sur, C. Badulescu, J.-D. Mathias. Using deconvolution to improve the metrological performance of the grid method. Optics and Lasers in Engineering, 51, 716-734, 2013

[10] A. Chrysochoos and Y. Surrel. Chapter 1. Basics of metrology and introduction to techniques. In M. Grédiac and F. Hild, editors, Full-field measurements and identification in solid mechanics, pp.1-29. Wiley, 2012.

[11] M. Grédiac, F. Sur, Effect of sensor noise on the resolution and spatial resolution of the displacement and strain maps obtained with the grid method, Strain, 50(1): 1-27, 2014

[12] S. Mallat. A Wavelet Tour of Signal Processing, Third Edition: The Sparse Way. Academic Press, third edition, 2008

[13] F. Hild, S. Roux. Comparison of local and global approaches to digital image correlation. Experimental Mechanics, 52(9):1503-1519, 2012.

[14] H. Haddadi, S. Belhabib. Use of a rigid-body motion for the investigation and estimation of the measurement errors related to digital image correlation technique. Optics and Lasers in Engineering, 46:185-196, 2008 